\title{
ON $\mathcal{M}$-HARMONIC BLOCH FUNCTIONS AND THEIR CARLESON MEASURES ${ }^{\dagger}$
}

\author{
by BOO RIM CHOE and YOUNG JOO LEE
}

(Received 20 November, 1996)

\begin{abstract}
On the setting of the unit ball of the complex $n$-space, some characterizations of $\mathcal{M}$-harmonic Bloch functions are obtained. As an application, Carleson measures are characterized by means of Berezin type integrals of $\mathcal{M}$-harmonic Bloch functions. As one may expect, these results carry over to $\mathcal{M}$-harmonic little Bloch functions and vanishing Carleson measures.
\end{abstract}

1. Introduction. Let $B$ be the unit ball of the complex $n$-space $C^{n}$ with boundary $S$. For $f \in C^{1}(B)$, let us define

$$
Q f(z)=\sup _{\zeta \in S} \frac{\mid<\nabla f(z), \bar{\zeta}>+\overline{<\nabla \bar{f}(z), \bar{\zeta}>\mid}}{\beta(z, \zeta)} \quad(z \in B),
$$

where $\beta$ is the Bergman metric on $B$ and $\nabla f$ is the complex gradient of $f$. Here, the notation $\langle z, w\rangle$ denotes the usual Hermitian inner product for points $z, w \in C^{n}$. It is known [4] that $Q$ is invariant under all automorphisms of $B$ in the sense that $Q(f \circ \varphi)=Q f \circ \varphi$ for all $\varphi \in \mathcal{A}$, the group of all automorphisms (i.e. biholomorphic self-maps) of $B$.

A function $u \in C^{2}(B)$ is called $\mathcal{M}$-harmonic on $B$ if it is annihilated on $B$ by the invariant Laplacian $\tilde{\Delta}$. See Section 2 for relevant definitions. The $\mathcal{M}$-harmonic Bloch space $M \mathcal{B}$ is the space of all $\mathcal{M}$-harmonic functions $f$ on $B$ for which

$$
\|f\|=\sup _{z \in B} Q f(z)<\infty
$$

and the $\mathcal{M}$-harmonic little Bloch space $M \mathcal{B}_{0}$ is the subspace of $M \mathcal{B}$, consisting of functions $f$ for which the additional boundary vanishing condition

$$
\lim _{|z| \rightarrow 1} Q f(z)=0
$$

holds. By the invariance of $Q$ under $\mathcal{A}$ we see that $\|f \circ \varphi\|=\|f\|$, for all $\varphi \in \mathcal{A}$.

If $f$ is holomorphic on $B$, it is known [10] that $f$ is a Bloch function if and only if $\left(1-|z|^{2}\right)|\nabla f(z)|=O(1)$ and $f$ is a little Bloch function if and only if $\left(1-|z|^{2}\right)|\nabla f(z)|=o(1)$. Many other conditions characterizing holomorphic (little) Bloch functions are well known. See, for example, [2], [3], [5], [9], [10], [11] and references therein. In the $\mathcal{M}$-harmonic case, Hahn and Youssfi [4] first studied and characterized $\mathcal{M}$-harmonic Bloch functions in terms of the Berezin transform, invariant Laplacian and BMO type integrals. Recently, Jevitć and Pavlović [6] have shown that many characterizations of holomorphic (little) Bloch functions also characterize $\mathcal{M}$-harmonic ones by giving characterizations in terms of various derivatives.

'This research is in part supported by $\operatorname{KOSEF}(97-0701-01-01-3), \operatorname{BSRI}(96-1407,1426)$, and GARC(97).

Glasgow Math. J. 40 (1998) 273-289. 
In the present paper, we add some other characterizations of $M B$ and $M B_{0}$. Our results imply that recent characterizations of Xiao and Zhong [12], [13] for holomorphic (little) Bloch functions (on the disc) continue to hold for $\mathcal{M}$-harmonic ones. To state our result, let $V$ denote the normalized Lebesgue volume measure on $B, \varphi_{a}$ be the standard automorphism of $B$ such that $\varphi_{a}(0)=a$, and write $d(z, w)$ for the Bergman distance between two points $z, w \in B$. For details, see Section 2.

Theorem A. Let $1 \leq p<\infty$. Then, for a function $f \mathcal{M}$-harmonic on $B$, the following statements are equivalent.

(a) $f \in M B$.

(b) $\sup _{\substack{z w w B \\ z \neq w}} \frac{|f(z)-f(w)|}{d(z, w)}<\infty$

(c) $\sup _{a \in B} \int_{B}\left|f \circ \varphi_{a}-f(a)\right|^{p} d V<\infty$

(d) $\sup _{a \in B} \int_{B}(Q f(z))^{p}\left(\frac{1-|a|^{2}}{|1-<z, a>|^{2}}\right)^{n+1} d V(z)<\infty$.

(e) There is a constant $t>0$ such that

$$
\sup _{a \in B} \int_{B} \exp \left(t\left|f \circ \varphi_{a}-f(a)\right|\right) d V<\infty
$$

Note that the condition (d) of Theorem A can be rephrased as "the Berezin transform of the measure $(Q f)^{p} d V$ is bounded". As is well known (see, for example, [14, Theorem A]), the Berezin transform of a positive Borel measure $\mu$ on $B$ is bounded if and only if $\mu$ is a Carleson measure. To be more precise, let $E_{r}(a)=\varphi_{a}(r B)$ denote the pseudohyperbolic ball with center $a \in B$ and radius $r \in(0,1)$. Then, $\mu$ is called a Carleson measure if

$$
\sup _{a \in B} \frac{\mu\left(E_{r}(a)\right)}{V\left(E_{r}(a)\right)}<\infty
$$

for some $r$. As an application of Theorem A, we prove the following theorem which characterizes Carleson measures by means of their action on Berezin type integrals of $\mathcal{M}$-harmonic Bloch functions.

THEOREM B. Let $0<p<\infty$. Then, a positive Borel measure $\mu$ on B is a Carleson measure if and only if there is a constant $C$ such that

$$
\sup _{a \in B} \int_{B}|f(z)-f(a)|^{p}\left(\frac{1-|a|^{2}}{|1-<z, a>|^{2}}\right)^{n+1} d \mu(z) \leq C\|f\|^{p},
$$

for all $f \in M B$. 
The equivalences of Theorem A carry over to $\mathcal{M}$-harmonic little Bloch functions.

THEOREM C. Let $1 \leq p<\infty$ and $0<r<1$. Then, for a function $f$ that is $\mathcal{M}$-harmonic on $B$, the following statements are equivalent.

(a) $f \in M \mathcal{B}_{0}$.

(b) $\lim _{|a| \rightarrow 1} \sup _{\substack{z \in E_{f}(a) \\ z \neq a}} \frac{|f(z)-f(a)|}{d(z, a)}=0$.

(c) $\lim _{|a| \rightarrow 1} \int_{B}^{z \neq a}\left|f \circ \varphi_{a}-f(a)\right|^{p} d V=0$.

(d) $\lim _{|a| \rightarrow 1} \int_{B}(Q f(z))^{p}\left(\frac{1-|a|^{2}}{|1-<z, a>|^{2}}\right)^{n+1} d V(z)=0$.

(e) There is a constant $t>0$ such that

$$
\lim _{|a| \rightarrow 1} \int_{B} \exp \left(t\left|f \circ \varphi_{a}-f(a)\right|\right) d V=1 .
$$

Also, the equivalence of Theorem B carries over to vanishing Carleson measures $\mu$ on $\mathrm{B}$ that satisfy

$$
\lim _{|a| \rightarrow 1} \frac{\mu\left(E_{r}(a)\right)}{V\left(E_{r}(a)\right)}=0
$$

for some $r$.

Theorem D. Let $0<p<\infty$. Then, a positive Borel measure $\mu$ on $B$ is a vanishing Carleson measure if and only if

$$
\lim _{|a| \rightarrow 1} \sup _{\substack{f \in M B \\ \mathbb{i}\{=1}} \int_{B}|f(z)-f(a)|^{p}\left(\frac{1-|a|^{2}}{|1-<z, a>|^{2}}\right)^{n+1} d \mu(z)=0 .
$$

In Section 2, we collect some notations and basic facts needed in the proofs. In Section 3, we prove Theorems $A$ and $C$. In fact, Theorem $A$ is restated and proved in the form of "quantity equivalence" with weights $\left(1-|z|^{2}\right)^{\alpha}$. Also, the corresponding weighted version of Theorem $C$ is proved. In Section 4, we first note the Carleson measure characterization of $\mathcal{M}$-harmonic (little) Bloch functions as a consequence of results obtained in the previous section. Then, as an application of results obtained in Section 3, we prove the weighted version of Theorem B in the form of "quantity equivalence". In the course of the proof, we notice that actions of Carleson measures on holomorphic or $\mathcal{M}$-harmonic Bloch functions make no difference in a certain sense (see Theorem 7). Also, we have the corresponding weighted version of Theorem $\mathrm{D}$.

2. Preliminaries. For $z \in B$, the standard automorphism $\varphi_{z}$ is given by

$$
\varphi_{z}(w)=\frac{z-P_{z} w-\sqrt{1-|z|^{2}} Q_{z} w}{1-<w, z>} \quad(w \in B)
$$


where $P_{z}$ denotes the orthogonal projection of $C^{n}$ onto the subspace generated by $z$ and $Q_{z}=I-P_{z}$. Then $\varphi_{z} \in \mathcal{A}, \varphi_{z}(0)=z$ and $\varphi_{z} \circ \varphi_{z}$ is the identity map on B. Furthermore, the real Jacobian $J_{R} \varphi_{z}$ of $\varphi_{z}$ is given by

$$
J_{R} \varphi_{z}(w)=\left(\frac{1-|z|^{2}}{11-<w, z>1^{2}}\right)^{n+1} \quad(w \in B)
$$

and the identity

$$
1-<\varphi_{z}(a), \varphi_{z}(b)>=\frac{\left(1-|z|^{2}\right)(1-<a, b>)}{(1-<a, z>)(1-<z, b>)}
$$

holds for every $a, b \in B$. See [7, Chapter 2] for details.

For $\alpha>-1$, define a measure $d V_{\alpha}$ on $B$ by $d V_{\alpha}(z)=\lambda_{\alpha}\left(1-|z|^{2}\right)^{\alpha} d V(z)$, where the constant $\lambda_{\alpha}$ is chosen so that $V_{\alpha}(B)=1$. For $a \in B$ and $\alpha>-1$, we put

$$
k_{a}^{\alpha}(z)=\left(\frac{\sqrt{1-|a|^{2}}}{1-<z, a>}\right)^{n+1+\alpha} \quad(z \in B)
$$

for notational simplicity. By (2) and (3), we have a useful change-of-variable formula:

$$
\int_{B} h(z) d V_{\alpha}(z)=\int_{B} h\left(\varphi_{a}(z)\right)\left|k_{a}^{\alpha}(z)\right|^{2} d V_{\alpha}(z) \quad(z \in B),
$$

for all measurable $h$ on $B$, whenever the integrals make sense.

For $u \in C^{2}(B)$, the invariant Laplacian $\tilde{\Delta} u$ is defined by

$$
(\tilde{\Delta} u)(z)=\Delta\left(u \circ \varphi_{z}\right)(0) \quad(z \in B)
$$

where $\Delta$ denotes the ordinary Laplacian. The operator $\tilde{\Delta}$ commutes with automorphisms in the sense that $\tilde{\Delta}(u \circ \varphi)=(\tilde{\Delta} u) \circ \varphi$, for all $\varphi \in \mathcal{A}$. Hence $\mathcal{M}$-harmonic functions are closed under composition with automorphisms. Moreover, by the invariant mean value property [7, Theorem 4.2.4] and a simple application of the integration in polar coordinates, we have the following mean value property for $\mathcal{M}$-harmonic functions $f$ :

$$
f(z)=\frac{1}{V_{\alpha}(r B)} \int_{r B} f \circ \varphi_{z} d V_{\alpha} \quad(z \in B, 0<r<1) .
$$
by

Given $z \in B$ and $\zeta \in C^{n}$, the Bergman metric $\beta(z, \zeta)$, modulo a constant factor, is given

$$
\beta(z, \zeta)=\left(\frac{\left(1-|z|^{2}\right)|\zeta|^{2}+|<z, \zeta>|^{2}}{\left(1-|z|^{2}\right)^{2}}\right)^{1 / 2}
$$


and the corresponding distance $d(z, w)$, called the Bergman distance, has the explicit formula

$$
d(z, w)=\frac{1}{2} \log \frac{1+\left|\varphi_{z}(w)\right|}{1-\left|\varphi_{z}(w)\right|} \quad(z, w \in B) .
$$

In particular, for any $0<p<\infty$ and $\alpha>-1$, the function $d^{p}(z, 0)$ is integrable with respect to the measure $d V_{\alpha}$. We note that

$$
\beta(z, \zeta) \leq \frac{|\zeta|}{1-|z|^{2}} \quad\left(z \in B, \zeta \in C^{n}\right)
$$

and the Bergman distance is invariant under $\mathcal{A}$. See Section 2 of [8] for details.

3. Characterizations of $M \mathcal{B}$ and $M \mathcal{B}_{0}$. We begin with a simple lemma.

Lemma 1. Let $f \in C^{1}(B)$. Then we have

$$
|f(z)-f(0)| \leq\left(\sup _{|w| \leq|z|} Q f(w)\right) d(0, z),
$$

for all $z \in B$.

Proof. We first note that by (6) we have

$$
\begin{aligned}
|f(z)-f(0)| & \left.=\mid \int_{0}^{1}\{<\nabla f(t z), \bar{z}\rangle+\overline{<\nabla \bar{f}(t z), \bar{z}>}\right\} d t \mid \\
& \leq \int_{0}^{1} \frac{|<\nabla f(t z), \bar{z} /| z \mid>+\overline{<\nabla \bar{f}(t z), \bar{z} /|z|>\mid}}{\beta(t z, z /|z|)}|z| \beta(t z, z /|z|) d t \\
& \leq \int_{0}^{1} \frac{Q f(t z)|z|}{1-|t z|^{2}} d t \\
& \leq\left(\sup _{|w| \leq|z|} Q f(w)\right) \int_{0}^{1} \frac{|z|}{1-|t z|^{2}} d t,
\end{aligned}
$$

for all $z \in B$. Since

$$
\int_{0}^{1} \frac{|z|}{1-|t z|^{2}} d t=\frac{1}{2} \log \frac{1+|z|}{1-|z|}=d(0, z),
$$

for all $z \in B$, we have the desired result. This completes the proof.

We are ready to characterize $\mathcal{M}$-harmonic Bloch functions. The equivalence of the quantities in (a) and (c) of the following theorem was proved in [4, Theorem 5.4] in the unweighted case of $\alpha=0$. 
THEOREM 2. Let $1 \leq p<\infty$ and $\alpha>-1$. Then the following quantities are equivalent as $f$ runs over all $\mathcal{M}$-harmonic functions on $B$ :

(a) $\|f\|$,

(b) $\|f\|_{b}=\sup _{\substack{z, w \in B \\ z \neq w}} \frac{|f(z)-f(w)|}{d(z, w)}$,

(c) $\|f\|_{c, p}=\sup _{a \in B}\left(\int_{B}\left|f \circ \varphi_{a}-f(a)\right|^{p} d V_{\alpha}\right)^{1 / p}$,

(d) $\|f\|_{d, p}=\sup _{a \in B}\left(\int_{B}(Q f)^{p}\left|k_{a}^{\alpha}\right|^{2} d V_{\alpha}\right)^{1 / p}$,

(e) $\|f\|_{e}=\inf _{t>0} \sup _{a \in B} \log \left(\int_{B} \exp \left(t\left|f \circ \varphi_{a}-f(a)\right|\right) d V_{\alpha}\right)^{1 / t}$.

In the rest of the paper, the same letter $C$ will denote various positive constants which may change from one occurrence to the next. While constants $C$ may depend on variables like $n, p, r, \alpha$ or some others, they will always be independent of functions, points or measures under consideration.

Proof. By Lemma 1,

$$
|f(z)-f(0)| \leq\|f\| d(0, z)
$$

for all $z \in B$. Replacing $f$ by $f \circ \varphi_{w}$ and then $z$ by $\varphi_{w}(z)$, we get, by the invariance of \|\| and $d$ under $\mathcal{A}$,

$$
|f(z)-f(w)| \leq\left\|f \circ \varphi_{w}\right\| d\left(0, \varphi_{w}(z)\right)=\|f\| d(z, w)
$$

for all $z, w \in B$, and so we have $\|f\|_{b} \leq\|f\|$.

Next, we show that $\|f\|_{c, p} \leq C\|f\|_{b}$. By the invariance of $d$ under $\mathcal{A}$, we see that

$$
\left|f \circ \varphi_{a}(z)-f(a)\right| \leq\|f\|_{b} d(z, 0),
$$

for all $z, a \in B$. It follows that

$$
\int_{B}\left|f \circ \varphi_{a}(z)-f(a)\right|^{p} d V_{\alpha} \leq\|f\|_{b}^{p} \int_{B} d^{p}(z, 0) d V_{\alpha}(z) \leq C\|f\|_{b}^{p}
$$

for all $a \in B$ and hence $\|f\|_{c, p} \leq C\|f\|_{b}$, as desired.

Next, we show $\|f\|_{d, p} \leq C\|f\|_{c, p}$. Assume that $\|f\|_{c, p}<\infty$. Then, by (5), with $r \rightarrow 1$ and the change-of-variable formula (4), one can see that

$$
f(z)=\int_{B} f \circ \varphi_{z} d V_{\alpha}=\int_{B} f(w)\left(\frac{1-|z|^{2}}{|1-<z, w>|^{2}}\right)^{n+1+\alpha} d V_{\alpha}(w) \quad(z \in B)
$$


Differentiation under the integral sign yields

$$
1<\nabla f(0), \bar{\zeta}>\left|\leq C \int_{B}\right| f \mid d V_{\alpha}
$$

and

$$
|<\nabla \bar{f}(0), \bar{\zeta}>| \leq C \int_{B}|f| d V_{\alpha}
$$

for all $\zeta \in S$. It follows from the definition of $Q$ and Jensen's inequality that

$$
Q f(0) \leq C \int_{B}|f| d V_{\alpha} \leq C\left(\int_{B}|f|^{p} d V_{\alpha}\right)^{1 / p} .
$$

Apply the above inequalities to $f \circ \varphi_{z}-f(z)$ to obtain

$$
Q f(z) \leq C\left(\int_{B}\left|f \circ \varphi_{z}-f(z)\right|^{p} d V_{\alpha}\right)^{1 / p},
$$

for all $z \in B$. Note that $k_{a}^{\alpha}$ has norm 1 in $L^{2}\left(d V_{\alpha}\right)$, for all $a \in B$, by (4). It follows from (7) that

$$
\begin{aligned}
\int_{B}(Q f)^{p}\left|k_{a}^{\alpha}\right|^{2} d V_{\alpha} & \leq C \int_{B} \int_{B}\left|f \circ \varphi_{z}-f(z)\right|^{p}\left|k_{a}^{\alpha}(z)\right|^{2} d V_{\alpha} d V_{\alpha}(z) \\
& \leq C\left(\sup _{z \in B} \int_{B}\left|f \circ \varphi_{z}-f(z)\right|^{p} d V_{\alpha}\right) \int_{B}\left|k_{a}^{\alpha}\right|^{2} d V_{\alpha} \\
& =C \sup _{z \in B} \int_{B}\left|f \circ \varphi_{z}-f(z)\right|^{p} d V_{\alpha},
\end{aligned}
$$

for all $a \in B$, and so we have $\|f\|_{d, p} \leq C\|f\|_{c, p}$.

Next, we show $\|f\| \leq C\|f\|_{d, p}$. Fix $r \in(0,1)$. By $(5)$, we have, for each $t \in(-1,1)$ and $\zeta \in S$,

$$
f(t \zeta)=\frac{1}{V_{\alpha}(r B)} \int_{r B} f \circ \varphi_{l \zeta} d V_{\alpha}
$$

Fixing $\zeta, w$ and denoting the $j$-th component of $\varphi_{t \zeta}(w)$ by $\varphi_{j}(t)$, one can see that

$$
\varphi_{j}^{\prime}(0)=\zeta_{j}-<w, \zeta>w_{j} \text { and } \bar{\varphi}_{j}^{\prime}(0)=\overline{\zeta_{j}-<w, \zeta>w_{j}}
$$

for each $j$. Thus,

$$
\left.\frac{d}{d t} f \circ \varphi_{t \zeta}(w)\right|_{t=0}=<\nabla f(w), \overline{\zeta-<w, \zeta>w}>+\overline{<\nabla \bar{f}(w), \overline{\zeta-<w, \zeta>w}>}
$$


for each $w \in B$ and $\zeta \in S$. It follows that

$$
\begin{aligned}
<\nabla & f(0), \bar{\zeta}>+\overline{<\nabla \bar{f}(0), \bar{\zeta}>} \\
& =\left.\frac{d}{d t} f(t \zeta)\right|_{t=0} \\
& =\left.\frac{1}{V_{\alpha}(r B)} \int_{r B} \frac{d}{d t} f \circ \varphi_{t \zeta}(w)\right|_{t=0} d V_{\alpha}(w) \\
& =\frac{1}{V_{\alpha}(r B)} \int_{r B}<\nabla f(w), \overline{\zeta-<w, \zeta>w}>+\overline{<\nabla \bar{f}(w), \overline{\zeta<w, \zeta>w}>} d V_{\alpha}(w) .
\end{aligned}
$$

Hence by (6), one obtains

$$
Q f(0) \leq C \int_{r B} \frac{Q f(w)}{1-|w|^{2}} d V_{\alpha}(w) \leq C \int_{B} Q f d V_{\alpha} .
$$

Now replace $f$ by $f \circ \varphi_{a}$. Then use Jensen's inequality and the change-of-variable formula (4) to see that

$$
Q f(a) \leq C\left(\int_{B}\left(Q f\left(\varphi_{a}\right)\right)^{p} d V_{\alpha}\right)^{1 / p}=C\left(\int_{B}(Q f)^{p}\left|k_{a}^{\alpha}\right|^{2} d V_{\alpha}\right)^{1 / p},
$$

for all $a \in B$, so that we get $\|f\| \leq C\|f\|_{d, p}$.

Consequently, $\|f\|,\|f\|_{b},\|f\|_{c, p}$ and $\|f\|_{d, p}$ are all equivalent for each $p$ with $1 \leq p<\infty$. Since $\|f\|$ is independent of $p$ and equivalent to $\|f\|_{c, p}$, for each $p$ in $[1, \infty)$, it is equivalent, in particular, to $\|f\|_{c, 1}$. Thus, in order to finish the proof, it is sufficient to prove the inequalities $\|f\|_{c, 1} \leq\|f\|_{e} \leq C\|f\|$.

By Lemma 1, we get as before

$$
\left|f \circ \varphi_{a}(z)-f(a)\right| \leq\|f\| d(z, 0)=\frac{\|f\|}{2} \log \frac{1+|z|}{1-|z|},
$$

for all $z, a \in B$. Assume $0<\|f\|<\infty$. Then, by taking $t=(\alpha+1) /\|f\|$, one can see from (9) that

$$
\begin{gathered}
\|f\|_{e} \leq \frac{\|f\|}{\alpha+1} \sup _{a \in B}\left(\log \int_{B} \exp \left(\frac{\alpha+1}{\|f\|}\left|f \circ \varphi_{a}-f(a)\right|\right) d V_{\alpha}\right) \\
\leq \frac{\|f\|}{\alpha+1} \log \int_{B}\left(\frac{1+|z|}{1-|z|}\right)^{\frac{\alpha+1}{2}} d V_{\alpha}(z) .
\end{gathered}
$$

Since the last integral above is finite, we have $\|f\|_{e} \leq C\|f\|$.

Finally, the inequality $\|f\|_{c, 1} \leq\|f\|_{e}$ is an easy consequence of Jensen's inequality. The proof is complete. 
As a result corresponding to Theorem 2, we characterize the $\mathcal{M}$-harmonic little Bloch space. In the following theorem, the equivalences of (a), (b) and (e) were proved for holomorphic functions on the disk in [13, Theorem 2.1] and the equivalence of (a) and (c) is given in [4, Theorem 5.6] in the unweighted case of $\alpha=0$.

TheOREM 3. Let $1 \leq p<\infty, \alpha>-1$ and $0<r<1$. Then the following statements are equivalent for a function $f$ that is $\mathcal{M}$-harmonic on $B$.

(a) $f \in M B_{0}$.

(b) $\lim _{|a| \rightarrow 1} \sup _{z \in E_{r}(a)} \frac{|f(z)-f(a)|}{d(z, a)}=0$.

(c) $\lim _{|a| \rightarrow 1} \int_{B}^{\substack{z \neq a \\ B}}\left|f \circ \varphi_{a}-f(a)\right|^{p} d V_{\alpha}=0$.

(d) $\lim _{|a| \rightarrow 1} \int_{B}(Q f)^{p}\left|k_{a}^{\alpha}\right|^{2} d V_{\alpha}=0$.

(e) There exists a constant $t>0$ such that

$$
\lim _{|a| \rightarrow 1} \int_{B} \exp \left(t\left|f \circ \varphi_{a}-f(a)\right|\right) d V_{\alpha}=1
$$

Before proceeding to the proof, we note that

$$
1-|w|^{2} \approx 1-|a|^{2} \quad\left(w \in E_{r}(a)\right)
$$

for each fixed $r \in(0,1)$. This follows from (3). Here and elsewhere, the notation $A(w) \approx B(a)$ means that two quantities have ratio bounded and bounded away from 0 by constants independent of the points $w, a$ under consideration.

Proof. We first prove the equivalence of (a) and (b). We shall assume (a) holds and prove (b). By Lemma 1, we have

$$
|f(z)-f(0)| \leq\left(\sup _{|w|<r} Q f(w)\right) d(0, z),
$$

for $|z|<r$. Replacing $f$ by $f \circ \varphi_{a}$ and, using the invariance of $Q$ under $\mathcal{A}$, one obtains

$$
\left|f \circ \varphi_{a}(z)-f(a)\right| \leq\left(\sup _{|w|<r} Q f\left(\varphi_{a}(w)\right)\right) d(0, z)
$$

for $|z|<r$. It follows from the invariance of $d$ under $\mathcal{A}$ that 


$$
\begin{aligned}
\sup _{\substack{z \in E_{r}(a) \\
z \neq a}} \frac{|f(z)-f(a)|}{d(z, a)} & =\sup _{0<|z|<r} \frac{\left|f \circ \varphi_{a}(z)-f(a)\right|}{d\left(\varphi_{a}(z), a\right)} \\
& =\sup _{0<|z|<r} \frac{\left|f \circ \varphi_{a}(z)-f(a)\right|}{d(z, 0)} \\
& \leq \sup _{|w|<r} Q f\left(\varphi_{a}(w)\right) \\
& =\sup _{w \in E_{r}(a)} Q f(w),
\end{aligned}
$$

for all $a \in B$. Now, letting $|a| \rightarrow 1$, we obtain (b) by (10).

Assume (b) holds. Using (5), one can easily see as before that

$$
Q f(0) \leq C \int_{r B}|f| d V .
$$

Replace $f$ by $f \circ \varphi_{a}-f(a)$ and then use the change-of-variable formula (4) to see that

$$
\begin{aligned}
Q f(a) & \leq C \int_{r B}\left|f \circ \varphi_{a}-f(a)\right| d V \\
& =C \int_{E_{r}(a)}|f(z)-f(a)| \frac{\left(1-|a|^{2}\right)^{n+1}}{|1-<z, a>|^{2 n+2}} d V(z) \\
& \leq C\left(\sup \frac{|f(z)-f(a)|}{d(z, a)}\right) \int_{E_{r}(a)} d(z, a) \frac{\left(1-|a|^{2}\right)^{n+1}}{|1-<z, a>|^{2 n+2}} d V(z) \\
& =C\left(\sup \frac{|f(z)-f(a)|}{d(z, a)}\right) \int_{r B} d(z, 0) d V(z) \\
& \leq C\left(\sup \frac{|f(z)-f(a)|}{d(z, a)}\right)
\end{aligned}
$$

for each $a \in B$, where sup is taken over all $z \in E_{r}(a)$ with $z \neq a$. Letting $|a| \rightarrow 1$, we have proved (a).

We assume (a) holds and prove (c). Let $a \in B$. Then, by (9) and the invariance of $d$ under $\mathcal{A}$, one obtains

$$
\begin{aligned}
\int_{B} \mid f & \circ \varphi_{a}-\left.f(a)\right|^{p} d V_{\alpha} \\
& =\int_{r B}\left|f \circ \varphi_{a}-f(a)\right|^{p} d V_{\alpha}+\int_{B \backslash r B}\left|f \circ \varphi_{a}-f(a)\right|^{p} d V_{\alpha} \\
& \leq\left(\sup _{0<|z|<r} \frac{\left|f \circ \varphi_{a}(z)-f(a)\right|}{d(z, 0)}\right)^{p} \int_{r B} d^{p}(z, 0) d V_{\alpha}(z)+\|f\|^{p} \int_{B \backslash r B} d^{p}(z, 0) d V_{\alpha}(z) \\
& \leq C\left(\sup _{\substack{z \in E_{r}(a) \\
z \neq a}} \frac{|f(z)-f(a)|}{d(z, a)}\right)^{p}+\|f\|^{p} \int_{B \backslash r B} d^{p}(z, 0) d V_{\alpha}(z) .
\end{aligned}
$$


Having seen that (a) and (b) are equivalent, one can see that the first term of the expression above tends to 0 as $|a| \rightarrow 1$, for each $r$. Consequently, first taking the limit as $|a| \rightarrow 1$ and then as $r \rightarrow 1$, we obtain (c).

Assume (c) and show (d). Note that $f \in M \mathcal{B}$ by Theorem 2. By (10), we have

$$
\lim _{|a| \rightarrow 1} \sup _{z \in E_{1}(a)} \int_{B}\left|f \circ \varphi_{z}-f(z)\right|^{p} d V_{\alpha}=0,
$$

for each $t \in(0,1)$. Now, by the change-of-variable formula (4) and (7), we have

$$
\begin{aligned}
\int_{B}(Q f)^{p}\left|k_{a}^{\alpha}\right|^{2} d V_{\alpha} & =\int_{t B}(Q f)^{p}\left(\varphi_{a}\right) d V_{\alpha}+\int_{B \backslash t B}(Q f)^{p}\left(\varphi_{a}\right) d V_{\alpha} \\
& \leq \sup _{z \in E_{t}(a)}(Q f)^{p}(z)+\|f\|^{p} V_{\alpha}(B \backslash t B) \\
& \leq C\left(\sup _{z \in E_{t}(a)} \int_{B}\left|f \circ \varphi_{z}-f(z)\right|^{p} d V_{\alpha}\right)+\|f\|^{p} V_{\alpha}(B \backslash t B)
\end{aligned}
$$

Consequently, first taking the limit as $|a| \rightarrow 1$ and then as $t \rightarrow 1$, we obtain (d) by (11).

The implication (d) $\Rightarrow$ (a) is a consequence of (8).

Consequently, (a), (b), (c) and (d) are all equivalent. Since (a) is independent of $p$ and equivalent to (c), for each $p$ in $[1, \infty)$, it is equivalent to (c) when $p=1$. Thus, in order to finish the proof, it is sufficient to show, (a) $\Rightarrow(\mathrm{e}) \Rightarrow$ (c) when $p=1$.

We assume (a) holds and prove (e). By Lemma 1 with $f \circ \varphi_{a}$ in place of $f$, we have

$$
\left|f \circ \varphi_{a}(z)-f(a)\right| \leq\left(\sup _{w \in E_{|z|}(a)} Q f(w)\right) d(0, z) \quad(z \in B) .
$$

Since $f \in M B_{0}$ by assumption, it follows from (10) that $\left|f \circ \varphi_{a}(z)-f(a)\right| \rightarrow 0$ as $|a| \rightarrow 1$, for each fixed $z \in B$. Choose $t>0$ such that $t\|f\|<2(\alpha+1)$. Then, by (12), one can see that

$$
\exp \left(t\left|f \circ \varphi_{a}(z)-f(a)\right|\right) \leq\left(\frac{1+|z|}{1-|z|}\right)^{\frac{|y| f \mid}{2}}
$$

for all $z, a \in B$. Since the right side of the above expression is integrable with respect to the measure $d V_{\alpha}$, (e) is a consequence of the Lebesgue dominated convergence theorem.

Finally, the implication (e) $\Rightarrow$ (c) with $p=1$ easily follows from Jensen's inequality. The proof is complete.

4. Carleson measures. Fix $\alpha>-1, r \in(0,1)$ and let $\mu$ be a positive Borel measure on $B$. We say that $\mu$ is an $\alpha$-weighted Carleson measure if

$$
\sup _{a \in B} \frac{\mu\left(E_{r}(a)\right)}{V_{\alpha}\left(E_{r}(a)\right)}<\infty
$$


If, in addition, $\mu$ satisfies the condition

$$
\lim _{|a| \rightarrow 1} \frac{\mu\left(E_{r}(a)\right)}{V_{\alpha}\left(E_{r}(a)\right)}=0
$$

we say that $\mu$ is an $\alpha$-weighted vanishing Carleson measure. It turns out that the notion of (vanishing) Carleson measures is independent of the choice of $r$. In fact, it is known (see for example, [14, Theorems A and B]) that $\mu$ is an $\alpha$-weighted Carleson measure if an only if its $\alpha$-weighted Berezin transform is bounded; that is

$$
\sup _{a \in B} \int_{B}\left|k_{a}^{\alpha}\right|^{2} d \mu<\infty .
$$

Similarly, $\mu$ is an $\alpha$-weighted vanishing Carleson measure if and only if

$$
\lim _{|a| \rightarrow 1} \int_{B}\left|k_{a}^{\alpha}\right|^{2} d \mu=0
$$

Hence the following corollary is an immediate consequence of Theorems 2 and 3.

Corollary 4. Let $1 \leq p<\infty, \alpha>-1$, and assume that $f$ is $\mathcal{M}$-harmonic on $B$.

(a) $f \in M \mathcal{B}$ if and only if $(Q f)^{p} d V_{\alpha}$ is an $\alpha$-weighted Carleson measure.

(b) $f \in M \mathcal{B}_{0}$ if and only if $(Q f)^{p} d V_{\alpha}$ is an $\alpha$-weighted vanishing Carleson measure. only if

It is also well known that, given $0<p<\infty, \mu$ is an $\alpha$-weighted Carleson measure if and

$$
\int_{B}|f|^{p} d \mu \leq C \int_{B}|f|^{p} d V_{\alpha},
$$

for all holomorphic functions $f$ in $L^{p}\left(d V_{\alpha}\right)$. In [12], Xiao observed that $\alpha$-weighted Carleson measures on the disc can be characterized by a similar integral condition, where $L^{p}$-integrals are replaced by Berezin type integrals of holomorphic Bloch functions. Here, we prove in Theorem 7 below that $\alpha$-weighted Carleson measures are also characterized by the same Berezin type integral condition for $\mathcal{M}$-harmonic Bloch functions. We first need a submean value type inequality for $\mathcal{M}$-harmonic functions.

Proposition 5. Let $0<p<\infty, 0<t<s<1$ and $\alpha>-1$. Then, there exists a constant C such that

$$
\sup _{z \in E_{1}(a)}|f(z)|^{p} \leq \frac{C}{V_{\alpha}\left(E_{s}(a)\right)} \int_{E_{s}(a)}|f|^{p} d V_{\alpha}
$$

for all $a \in B$ and $f$ an $\mathcal{M}$-harmonic function on $B$.

Before proceeding to the proof, we first note that, for a given $r$, we have

$$
V_{\alpha}\left(E_{r}(a)\right) \approx\left(1-|a|^{2}\right)^{n+1+\alpha} \quad(a \in B)
$$


Proof. Fix a point $a \in B$ and an $\mathcal{M}$-harmonic $f$. Let $z \in E_{i}(a)$ and $r=s-t$. Note that $E_{r}(z) \subset E_{s}(a)$ and hence $1-|w|^{2} \approx 1-|a|^{2}$, for all $w \in E_{r}(z)$, by (10). By Proposition 10.1 of [8] and (14), we have

$$
\begin{aligned}
|f(z)|^{p} & \leq C \int_{E_{r}(z)} \frac{|f(w)|^{p}}{\left(1-|w|^{2}\right)^{n+1+\alpha}} d V_{\alpha}(w) \\
& \leq \frac{C}{\left(1-|a|^{2}\right)^{n+1+\alpha}} \int_{E_{s}(a)}|f|^{p} d V_{\alpha} \\
& \leq \frac{C}{V_{\alpha}\left(E_{s}(a)\right)} \int_{E_{s}(a)}|f|^{p} d V_{\alpha},
\end{aligned}
$$

which completes the proof.

Before turning to Theorem 7, we need a simple lemma.

Lemma 6. For every $a, b$ and $w$ in $B$, we have

$$
\frac{1-\left|\varphi_{a}(b)\right|^{2}}{1-<\varphi_{a}(w), \varphi_{a}(b)>}=1-<\varphi_{b}(w), \varphi_{b}(a)>\text {. }
$$

Proof. A direct calculation by (3) completes the proof.

In the following the notation $\mathcal{B}$ denotes the holomorphic Bloch space.

Theorem 7. Let $0<p<\infty, 0<r<1$ and $\alpha>-1$. Then the following quantities are equivalent as $\mu$ runs over all positive Borel measures on $B$.

(a) $\|\mu\|_{a, p}=\sup _{a \in B} \sup _{\substack{f \in M B \\\|\wedge\|=1}} \int_{B}|f-f(a)|^{p}\left|k_{a}^{\alpha}\right|^{2} d \mu$.
(b) $\|\mu\|_{b, p}=\sup _{a \in B} \sup _{\substack{f \in \mathbb{B} \\ \mathbb{i} f=1}} \int_{B}|f-f(a)|^{p}\left|k_{a}^{\alpha}\right|^{2} d \mu$.
(c) $\|\mu\|_{c, r}=\sup _{a \in B} \frac{\mu\left(E_{r}(a)\right)}{V_{\alpha}\left(E_{r}(a)\right)}$.

Proof. The inequality $\|\mu\|_{b, p} \leq\|\mu\|_{a, p}$ is clear because $\mathcal{B} \subset M \mathcal{B}$.

Next, we show that $\|\mu\|_{c, r} \leq C\|\mu\|_{b, p}$. Let $t=(1+r) / 2$. Corresponding to each $a=|a| \zeta$ in $B, \zeta \in S$, let $b=-t \zeta$ and put

$$
f_{a}(z)=\frac{1}{1-<z, a_{0}>}, a_{0}=\varphi_{a}(b) \quad(z \in B) .
$$

Note that $a_{0} \neq 0$. Since $f_{a}$ is holomorphic, we have from [10] that

$$
\left\|f_{a}\right\| \approx \sup _{z \in B}\left(1-|z|^{2}\right)\left|\nabla f_{a}(z)\right|
$$


and therefore one can see from (3) that

$$
\left\|f_{a}\right\| \approx \sup _{z \in B} \frac{\left|a_{0}\right|\left(1-|z|^{2}\right)}{\left|1-<z, a_{0}>\right|^{2}}=\sup _{z \in B} \frac{\left|a_{0}\right|\left(1-\left|\varphi_{a_{0}}(z)\right|^{2}\right)}{1-\left|a_{0}\right|^{2}}=\frac{\left|a_{0}\right|}{\left(1-\left|a_{0}\right|^{2}\right)}
$$

Also, by (3), one can easily verify that

$$
1-\left|a_{0}\right|^{2} \approx 1-|a|^{2} \approx \mid 1-<z, a_{0}>1 \quad\left(z \in E_{r}(a)\right)
$$

Thus, it follows from (14) that

$$
\begin{aligned}
\|\mu\|_{b, p} & \geq \frac{1}{\left\|f_{a}\right\|^{p}} \int_{E_{r}(a)}\left|f_{a}(z)-f_{a}\left(a_{0}\right)\right|^{p}\left|k_{a_{o}}^{\alpha}(z)\right|^{2} d \mu(z) \\
& \geq \frac{C}{V_{\alpha}\left(E_{r}(a)\right)} \int_{E_{r}(a)}\left(\frac{1}{\left|a_{0}\right|}\left|1-\frac{1-\left|a_{0}\right|^{2}}{1-<z, a_{0}>}\right|\right)^{p} d \mu(z) .
\end{aligned}
$$

On the other hand, using the explicit formula (1) of the standard automorphism and simple manipulations, one can easily see that

$$
\varphi_{b}(a)=-\left(\frac{t+|a|}{1+t|a|}\right) \zeta
$$

and hence that

$$
\frac{1}{\left|\varphi_{b}(a)\right|}\left|<\varphi_{b}(w), \varphi_{b}(a)>1=\right|<\varphi_{b}(w), \zeta>1=\left|\frac{t+<w, \zeta>}{1+t<w, \zeta>}\right|,
$$

for all $w \in B$. Note from (3) that $\left|\varphi_{z}(w)\right|=\left|\varphi_{w}(z)\right|$, for all $z, w \in B$. Hence, it follows from Lemma 6 that

$$
\begin{aligned}
\inf _{z \in E_{r}(a)} \frac{1}{\left|a_{0}\right|}\left|1-\frac{1-\left|a_{0}\right|^{2}}{1-<z, a_{0}>}\right| & =\inf _{|w|<r} \frac{1}{\left|\varphi_{a}(b)\right|}\left|1-\frac{1-\left|\varphi_{a}(b)\right|^{2}}{1-<\varphi_{a}(w), \varphi_{a}(b)>}\right| \\
& =\inf _{|w|<r} \frac{1}{\left|\varphi_{b}(a)\right|} \mid<\varphi_{b}(w), \varphi_{b}(a)>1 \\
& =\inf _{|w|<r}\left|\frac{t+<w, \zeta>}{1+t<w, \zeta>}\right| \\
& \geq \frac{1-r}{4} .
\end{aligned}
$$

Combining the above with (15), we have

$$
\sup _{a \in B} \frac{\mu\left(E_{r}(a)\right)}{V_{\alpha}\left(E_{r}(a)\right)} \leq C\|\mu\|_{b, p},
$$

as desired. 
Finally, we show that $\|\mu\|_{a, p} \leq C\|\mu\|_{c, r}$. Using the same method of Axler [1, Lemma 3.5], we can choose a sequence $\left\{w_{i}\right\}$ of points in $B$ and a positive integer $M$ such that $\bigcup_{i=1}^{\infty} E_{r}\left(w_{i}\right)=B$ and each $z \in B$ is in at most $M$ of the sets $E_{(1+r) / 2}\left(w_{i}\right)$. Let $a \in B$ and $f \in M \mathcal{B}$ with $\|f\|=1$. Note that

$$
1-\left|\varphi_{a}(z)\right|^{2} \approx 1-\left|\varphi_{a}(w)\right|^{2}, \quad 1-|z|^{2} \approx 1-|w|^{2},
$$

for $z \in E_{l}(w)$ and $a \in B$ by (10). It follows from (3) that, for each fixed $l \in(0,1)$, $\left|k_{a}^{\alpha}(z)\right| \approx\left|k_{a}^{\alpha}(w)\right|$, for $z \in E_{l}(w)$ and $a \in B$. Thus we obtain from Proposition 5 , with $t=r$ and $s=(1+r) / 2$, that

$$
\begin{aligned}
\int_{B}|f-f(a)|^{p}\left|k_{a}^{\alpha}\right|^{2} d \mu & \leq \sum_{i=1}^{\infty} \int_{E_{r}\left(w_{i}\right)}|f-f(a)|^{p}\left|k_{a}^{\alpha}\right|^{2} d \mu \\
& \leq C \sum_{i=1}^{\infty}\left(\sup _{z \in E_{r}\left(w_{i}\right)}|f(z)-f(a)|^{p}\right)\left|k_{a}^{\alpha}\left(w_{i}\right)\right|^{2} \mu\left(E_{r}\left(w_{i}\right)\right) \\
& \leq C \sum_{i=1}^{\infty} \frac{\mu\left(E_{r}\left(w_{i}\right)\right)\left|k_{a}^{\alpha}\left(w_{i}\right)\right|^{2}}{V_{\alpha}\left(E_{s}\left(w_{i}\right)\right)} \int_{E_{s}\left(w_{i}\right)}|f-f(a)|^{p} d V_{\alpha} \\
& \leq C \sum_{i=1}^{\infty} \frac{\mu\left(E_{r}\left(w_{i}\right)\right)}{V_{\alpha}\left(E_{r}\left(w_{i}\right)\right)} \int_{E_{s}\left(w_{i}\right)}|f-f(a)|^{p}\left|k_{a}^{\alpha}\right|^{2} d V_{\alpha} \\
& \leq C\|\mu\|_{c, r} \sum_{i=1}^{\infty} \int_{E_{s}\left(w_{i}\right)}|f-f(a)|^{p}\left|k_{a}^{\alpha}\right|^{2} d V_{\alpha} \\
& \leq C M\|\mu\|_{c, r} \int_{B}\left|f \circ \varphi_{a}-f(a)\right|^{p} d V_{\alpha} .
\end{aligned}
$$

Thus, for $1 \leq p<\infty$, the desired inequality follows from Theorem 2 . For $0<p<1$, an application of Jensen's inequality shows that the last integral of the expression above is less than or equal to

$$
\sup _{a \in B}\left(\int_{B}\left|f \circ \varphi_{a}-f(a)\right| d V_{\alpha}\right)^{p} \approx\|f\|^{p}=1,
$$

by Theorem 2 again. The proof is complete.

Also, a slight modification of the above proof gives a corresponding result for $\alpha$-weighted vanishing Carleson measures as follows.

THEOREM 8. Let $0<p<\infty$ and $\alpha>-1$. Then the following statements are equivalent for a positive Borel measure $\mu$ on $B$.
(a) $\lim _{|a| \rightarrow 1} \sup _{\substack{f \in M B \\ \mathbb{f} f \|=1}} \int_{B}|f-f(a)|^{p}\left|k_{a}^{\alpha}\right|^{2} d \mu=0$.
(b) $\lim _{|a| \rightarrow 1} \sup _{\substack{f \in \mathcal{B} \\ \mathbb{f} f \mathfrak{l}=1}} \int_{B}|f-f(a)|^{p}\left|k_{a}^{\alpha}\right|^{2} d \mu=0$.
(c) $\mu$ is an $\alpha$-weighted vanishing Carleson measure. 
Proof. A trivial modification of the proof of Theorem 7 yields the implications (a) $\Rightarrow$ (b) $\Rightarrow(c)$. Now, we assume (c) holds and prove (a). Let $\left\{w_{i}\right\}$ be the sequence chosen in the proof of Theorem 7. Note that $\left|w_{i}\right| \rightarrow 1$ as $i \rightarrow \infty$. Since $\mu\left(E_{r}(a)\right) / V_{\alpha}\left(E_{r}(a)\right)$ tends to 0 as $|a| \rightarrow 1$, by assumption, for any $\epsilon>0$ there is a positive integer $N$ such that

$$
\frac{\mu\left(E_{r}\left(w_{i}\right)\right)}{V_{\alpha}\left(E_{r}\left(w_{i}\right)\right)}<\epsilon \quad(i>N)
$$

Let $a \in B$ and $f \in M B,\|f\|=1$. By an argument similar to the proof of Theorem 7 , one can see by Hölder's inequality that

$$
\begin{aligned}
& \sum_{i=1}^{N} \int_{E_{r}\left(w_{i}\right)}|f-f(a)|^{p}\left|k_{a}^{\alpha}\right|^{2} d \mu \\
& \quad \leq \sum_{i=1}^{N}\left(\int_{E_{r}\left(w_{i}\right)}\left|k_{a}^{\alpha}\right|^{2} d \mu\right)^{1 / 2}\left(\int_{E_{r}\left(w_{i}\right)}|f-f(a)|^{2 p}\left|k_{a}^{\alpha}\right|^{2} d \mu\right)^{1 / 2} \\
& \quad \leq C\left(\int_{B}\left|k_{a}^{\alpha}\right|^{2} d \mu\right)^{1 / 2}\left(\int_{B}\left|f \circ \varphi_{a}-f(a)\right|^{2 p} d V_{\alpha}\right)^{1 / 2} \sum_{i=1}^{N}\left(\frac{\mu\left(E_{r}\left(w_{i}\right)\right)}{V_{\alpha}\left(E_{r}\left(w_{i}\right)\right)}\right)^{1 / 2} \\
& \quad \leq C\left(\int_{B}\left|k_{a}^{\alpha}\right|^{2} d \mu\right)^{1 / 2} \sum_{i=1}^{N}\left(\frac{\mu\left(E_{r}\left(w_{i}\right)\right)}{V_{\alpha}\left(E_{r}\left(w_{i}\right)\right)}\right)^{1 / 2}
\end{aligned}
$$

and from (16), if we set $2 s=1+r$, then

$$
\begin{aligned}
\sum_{i=N+1}^{\infty} & \int_{E_{r}\left(w_{i}\right)}|f-f(a)|^{p}\left|k_{a}^{\alpha}\right|^{2} d \mu \\
& \leq C \sum_{i=N+1}^{\infty} \frac{\mu\left(E_{r}\left(w_{i}\right)\right)}{V_{\alpha}\left(E_{r}\left(w_{i}\right)\right)} \int_{E_{s}\left(w_{i}\right)}|f-f(a)|^{p}\left|k_{a}^{\alpha}\right|^{2} d V_{\alpha} \\
& \leq C M \epsilon \int_{B}\left|f \circ \varphi_{a}-f(a)\right|^{p} d V_{\alpha} \\
& \leq C M \epsilon .
\end{aligned}
$$

Consequently,

$$
\int_{B}|f-f(a)|^{p}\left|k_{a}^{\alpha}\right|^{2} d \mu \leq C\left(\int_{B}\left|k_{a}^{\alpha}\right|^{2} d \mu\right)^{1 / 2} \sum_{i=1}^{N}\left(\frac{\mu\left(E_{r}\left(w_{i}\right)\right)}{V_{\alpha}\left(E_{r}\left(w_{i}\right)\right)}\right)^{1 / 2}+C M \epsilon
$$

for each $a \in B$. Now, since $\epsilon>0$ is arbitrary, letting $|a| \rightarrow 1$, we get (a) by (13). The proof is complete 


\section{REFERENCES}

1. S. Axler, Bergman spaces and their operators in Surveys of some recent results in operator theory, Pitman Research Notes in Math. 1 (1988), 1-50.

2. S. Axler, The Bergman space, the Bloch space, and commutators of multiplication operators, Duke Math. J, 53 (1986), 315-332.

3. B. R. Choe, Projections, the weighted Bergman spaces, and the Bloch space, Proc. Amer. Math. Soc. 108 (1990), 127-136.

4. K. T. Hahn and E. H. Youssfi, $\mathcal{M}$-Harmonic Besov p-spaces and Hankel operators in the Bergman space on the unit ball in $C^{n}$, Manuscripta Math. 71 (1991), 67-81.

5. K. T. Hahn and E. H. Youssfi, Möbius invariant Besov p-spaces and Hankel operators in the Bergman space on the unit ball in $C^{n}$, Complex Variables 17 (1991), 89-104.

$1385-1392$

6. M. Jevitć and M. Pavlović, On M-harmonic Bloch space, Proc. Amer. Math. Soc. 123 (1995),

7. W. Rudin, Function theory in the unit ball of $C^{n}$ (Springer-Verlag, 1980).

8. M. Stoll, Invariant potential theory in the unit ball of $C^{n}$ (Cambridge University Press, 1994).

9. K. Stroethoff, Besov-type characterizations for the Bloch space, Bull. Aust. Math. Soc. 39 (1989), 405-420.

10. R. M. Timoney, Bloch functions in several complex variables I, Bull. London Math. Soc. 12 (1980), 241-264.

11. R. M. Timoney, Bloch functions in several complex variables II, J. Reine Angew. Math. 319 (1980), 1-22.

12. J. Xiao, Carleson measure, atomic decomposition and free interpolation from Bloch space, Ann. Acad. Sci. Ser. A. I. Math. 19 (1994), 35-46.

13. J. Xiao and L. Zhong, On little Bloch space, its Carleson measure, atomic decomposition and free interpolation, Complex Variables 27 (1995), 175-184,

14. K. Zhu, Positive Toeplitz operators on weighted Bergman spaces of bounded symmetric domains, J. Operator Theory 20 (1988), 329-357.

Boe Rim Choe

Department of Mathematics

KOREA UNIVERSITY

SEOUL $136-701$

KOREA

E-mail address: choebr@semi.korea.ac.kr
Young Joo Lee

Department of Mathematics

MOKPo National University

Chonnam 534-729

KOREA

E-mail address: yjlee@chungkye.mokpo.ac.kr 